\title{
Neural Clouds for Monitoring of Complex Systems
}

\author{
B. Lang ${ }^{a}$, T. Poppe ${ }^{b}$, A. Minin $c$, I. Mokhov ${ }^{a}$, Y. Kuperin ${ }^{c}$, A. Mekler ${ }^{d}$, and I. Liapakina ${ }^{e}$ \\ ${ }^{a}$ OOO Siemens, Fault Analysis and Prevention group \\ ${ }^{b}$ Siemens AG, Industry sector, Thomas.Poppe@siemens.com \\ ${ }^{c}$ Saint-Petersburg State University, \\ ${ }^{d}$ Institute of Human Brain RAS, \\ e Saint Petersburg State University of Economics and Finance \\ e-mail: \{Bernhard.Lang, Ilya.Mokhov\}@siemens.com; \{Alexey.Minin,Yuri.Kuperin\}@gmail.com; \\ mekler@yandex.ru; leonikaspb@gmail.com \\ Received April 21, 2008; in final form June 24, 2008
}

\begin{abstract}
Condition monitoring is an important and challenging task actual for many areas of industry, medicine and economics. Nowadays it is necessary to provide on-line monitoring of the complex systems status, e.g. the steel production, in order to avoid faults, breakdowns or wrong diagnostics. In the present paper a novel machine learning method for the automated condition monitoring is presented. Neural Clouds (NC) is a novel data encapsulation method, which provides a confidence measure regarding classification of the complex system conditions. The presented adaptive algorithm requires only the data which corresponds to the normal system conditions, which is typically available. At the same time the fault related data acquisition is expensive and fault modeling is not always possible, especially in case one is dealing with steel production, power stations operation, human health condition or critical phenomena in financial markets. These real word applications are also presented in the paper.
\end{abstract}

Key words: neural clouds, one side classification, on line monitoring, early fault detection, EEG classification, fault analysis and prevention.

DOI: $10.3103 / \mathrm{S} 1060992 \mathrm{X} 08030016$

\section{INTRODUCTION}

The Neural Clouds (NC) concept was successfully elaborated and applied by the Corporate Technology Department of Siemens AG for solving the steel production optimization problem [3]. However this technique has been also transferred, with the necessary modifications, to the field of vibration analysis [4], EEG classification and financial market analysis and prediction. The application of neuro-fuzzy methods, presented in this paper, is an attempt to make the expert condition monitoring system more intelligent and able to face the real world problems, keeping the monitoring costs reasonable.

The concept presented in the paper is directed for the elaboration an efficient data encapsulation method for the adaptive solving of the so called one-side classification problem. The basic idea behind the usage of the one-side classification in the field of condition monitoring and fault analysis is that the real data, which can be collected, usually corresponds to the normal conditions of the complex systems in question. Vice versa data collection, corresponding to abnormal conditions, is expensive, and fault modeling is not always available. Here one should mention that the idea of elaboration of the classifier based on a data, collected from the system under normal operating conditions, could be extended for the case of the human healthrelated measurements classification, where the data acquisition related to particular disease is even more problematic.

The NC is applied in the following fields: analysis of vibration data [11-12], electroencephalography [12, 5-9] and prediction of the American stock market declines [20-25].

\section{THE BASIS OF THE NEURAL CLOUDS ALGORITHM}

In this section the Neural Clouds method is presented. Let us assume that we are dealing with a number of measurements from a real object. Every instance of the given data set could be considered as a point in $\mathrm{n}$-dimensional space, where $\mathrm{n}$ corresponds to the number of different parameters of the system under consideration. First, the data set should be clustered. In most of the cases data should be normalized prior to the 


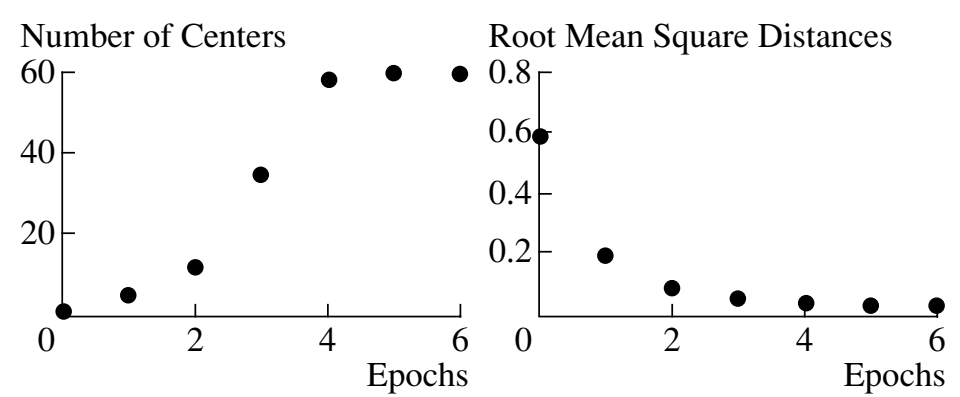

Fig. 1. Visualization of the AKM convergence.

clustering. Then, the data around the clusters have to be encapsulated with the so-called Gaussian bells. At next stage the normalization and summation of the mentioned Gaussian bells should be performed, in order to obtain a hyper-surface in the system variables space which will encapsulate all the data.

In the following a more detailed explanation of mentioned steps of the algorithm is provided.

\section{DATA NORMALIZATION}

As a first step the data normalization procedure should be performed in order to avoid clustering problems, corresponding to the possible significant difference in data distribution. The Min-Max normalization procedure was chosen, where the minimal value corresponds to minus identity and maximum value to plus identity respectively. An alternative approach is the normalization of the input data assuming a normal distribution diverse input data scaling is a way to modify the sensitivity per dimension. This will effect the calculation of the multi-dimensional distances for clustering purposes as well as the sensitivity of the final Neu- 1 ral Clouds regarding deviations from "normal" conditions.

\section{CLUSTERING}

The positioning of the radial nodes over the input space in such a way, when they represent groups or clusters of inputs vectors, can be carried out by means of any non-supervised algorithm for clustering. In our approach the well known K-Means algorithm [3-4] is adopted and an advanced variation of the KMeans algorithm is proposed (further AKM). The most remarkable enhancement introduced is that the algorithm is able to set the optimal number of centroids between a maximal and a minimal bounds defined by 2 the user. AKM sets centroids progressively according to density and volumetric distribution.

AKM itself consists of the next steps:

Set an initial number of centroids $n_{h}$, a maximal and a minimal bound. 2

Call k-means algorithm to position $n_{h}$ centroids. 2

Insert or erase centroids according to the following premises: 2

If the distances of data are above a certain distance from the nearest centroid, then generate a new centre. 3

If any cluster consists of less than a certain number of data, then remove the corresponding centroid.

If the distance between some centroids is smaller than a certain value, then combine those clusters to one. 2

Loop to step 2 unless a certain number of epochs is reached, or centroids number and their coordinates 2 became stable.

AKM algorithm visualization convergence is presented at Fig. 1.

\section{APPLICATION OF GAUSSIAN BELLS}

After all centroids have been extracted from the input data, the data should be encapsulated with the 2 hyper surface. For this purpose Gaussian distributions_-so called "Gaussian bells"—are used [2]:

$$
R_{i}(x)=e^{-\frac{\left|x-m_{i}\right|^{2}}{2 \sigma^{2}}},
$$

where $m_{i}$ is the centre of the Gaussian bell and $\sigma$ is the width of the Gaussian bell. The centroid of the AKM 3 cluster becomes the center of corresponding Gaussian bell. Each cluster is covered with the corresponding 


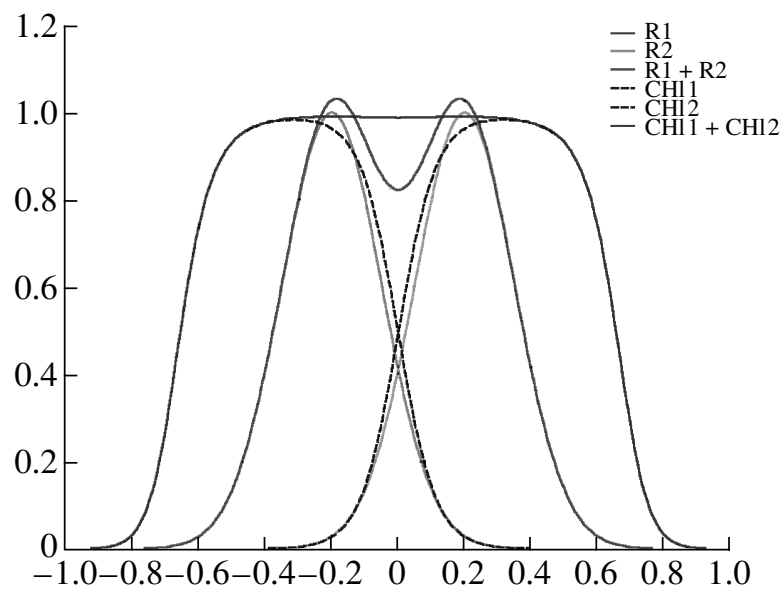

Fig. 2. Normalization effects with $g_{0}$. The influence of $g_{0}$ is discussed below.

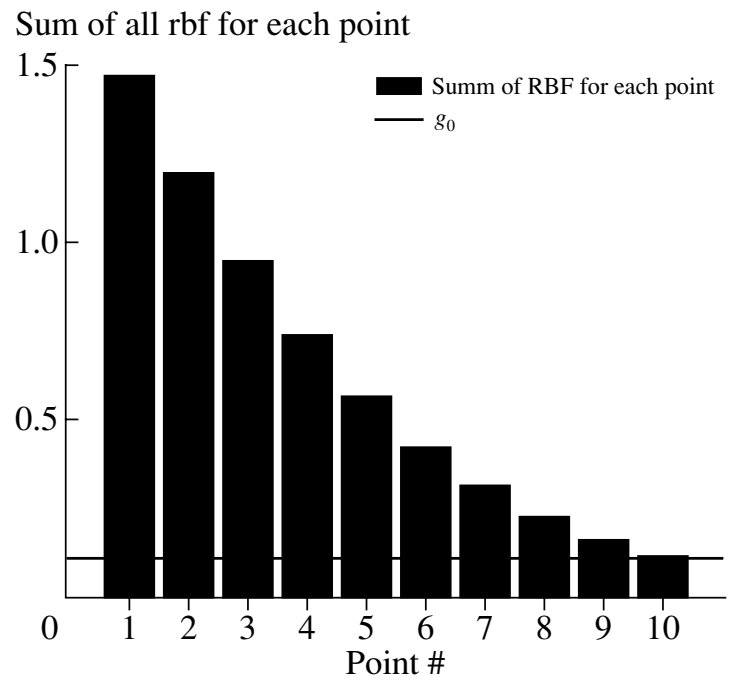

Fig. 3. Robust selection of the normalization factor $g_{0}$.

Gaussian bell. A constant width per dimension is selected in order to avoid side-effects, related to the subsequent normalization.

\section{NORMALIZATION OF THE GAUSSIAN BELLS}

The sum of all Gaussian bells is provided in order to obtain the encapsulating surface. It may be more than unity-in case these bells have cross sections (see Fig. 2). Therefore, normalization similar to the partitioning-to-one known for radial-basis function networks should be performed as far as those values would be used as probability measures with values between 0 and 1 . Applying the partitioning-to-one approach, however, leads to the value 1 overall the input space. An additional factor $g_{0}$ is introduced (see Eq. (2)).

As a first step of the Gaussian bells normalization let us perform the data outliers determination. For this purpose let us construct the supplemental Gaussian bells for every data point from the given set. Summing up the values of all Gaussian bells for given data set, values represented in Fig. 3 could be obtained. This information enables to determine the bias factor $g_{0}$, represented by the horizontal line, e.g. by setting it equal to the value, which corresponds to the first percentile of all sums of Gaussian bells (or sums of values of Radial Basis Functions (further RBF)). The underlying assumption is that the encapsulation of outliers should be avoided in order to obtain plausible results for the Neural Clouds. 


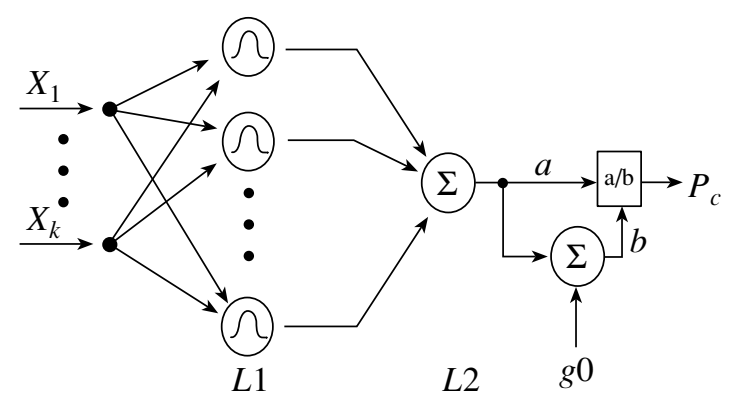

Fig. 4. Radial Basis Function network representation. L1 and L2 are Layer one and Layer two respectively. $X_{1 . . k}$ are input patterns and $P_{c}$ is a confidence value for the concrete pattern $X_{1 . . k}$.

The normalized data encapsulator is defined as:

$$
P_{c}(\mathbf{x})=\frac{\sum_{i=1}^{n} R_{i}(\mathbf{x})}{\sum_{i=1}^{n} R_{i}(\mathbf{x})+g_{0}},
$$

where $R_{i}(\mathbf{x})$ are the Gaussian bells, as defined by (1), and $g_{0}$ is a global factor, described above. Figure 3 shows the $g_{0}$ influence to the final width of the encapsulator. Given input $x$ as a matrix of inputs (patterns) 4 and the output will be a vector of confidence levels, calculated for each element.

Different sets of parameters $g_{0}, \sigma$ and the number of AKM clusters $n$ are responsible for the adaptation of the NC to the given data set and determining different tolerance levels. As a possible extension of the presented approach authors consider the automation of the mentioned above values selection. However, not all information for these parameters could be extracted out of the given data set. Sensitivity per dimension, the noise level in the data and the evidence of outliers in the data is critical information, usually provided by experts.

Here, it should be mentioned, that this constructed mechanism could be described in a form of Radial Basis Function network [10], as it is shown in Fig. 4. After such a network is manually trained by tuning mentioned parameters on the measured data, it could be used for the confidence level estimation for the new measurements.

The output vector, generated by the NC algorithm, is in a form of a confidence value between 0 and 1 that can be interpreted as a measure of the failure probability according to the expression in Eq. (3):

$$
P_{f}=\left|1-P_{c}\right|,
$$

where $P_{f}$ is failure probability and $P_{c}$ is a confidence level.

The visualization of the constructed sample NC is presented at Fig. 5. On the left side the confidence levels are represented by the contour lines and on the right—by the 3D surface.

\section{COMPARISON OF THE NC WITH PARZEN WINDOW AND GAUSSIAN MIXTURE ALGORITHMS}

In order to compare Neural Clouds with the same type algorithms authors have used Gaussian mixture approach [18] and Parzen window (Kernel density estimation) [19]. The comparison results could be found at Fig. 5. The dataset, consisting of 3 clusters, was artificially generated in order to compare different approaches (random number generator was used to create dataset).

As one can see Neural Cloud has a big advantage in comparison with other techniques since it creates unique plateau, which height could be interpreted as probability. If the measurement corresponds to the top of the plateau (see Eq. (3)), probability is equal to one. 

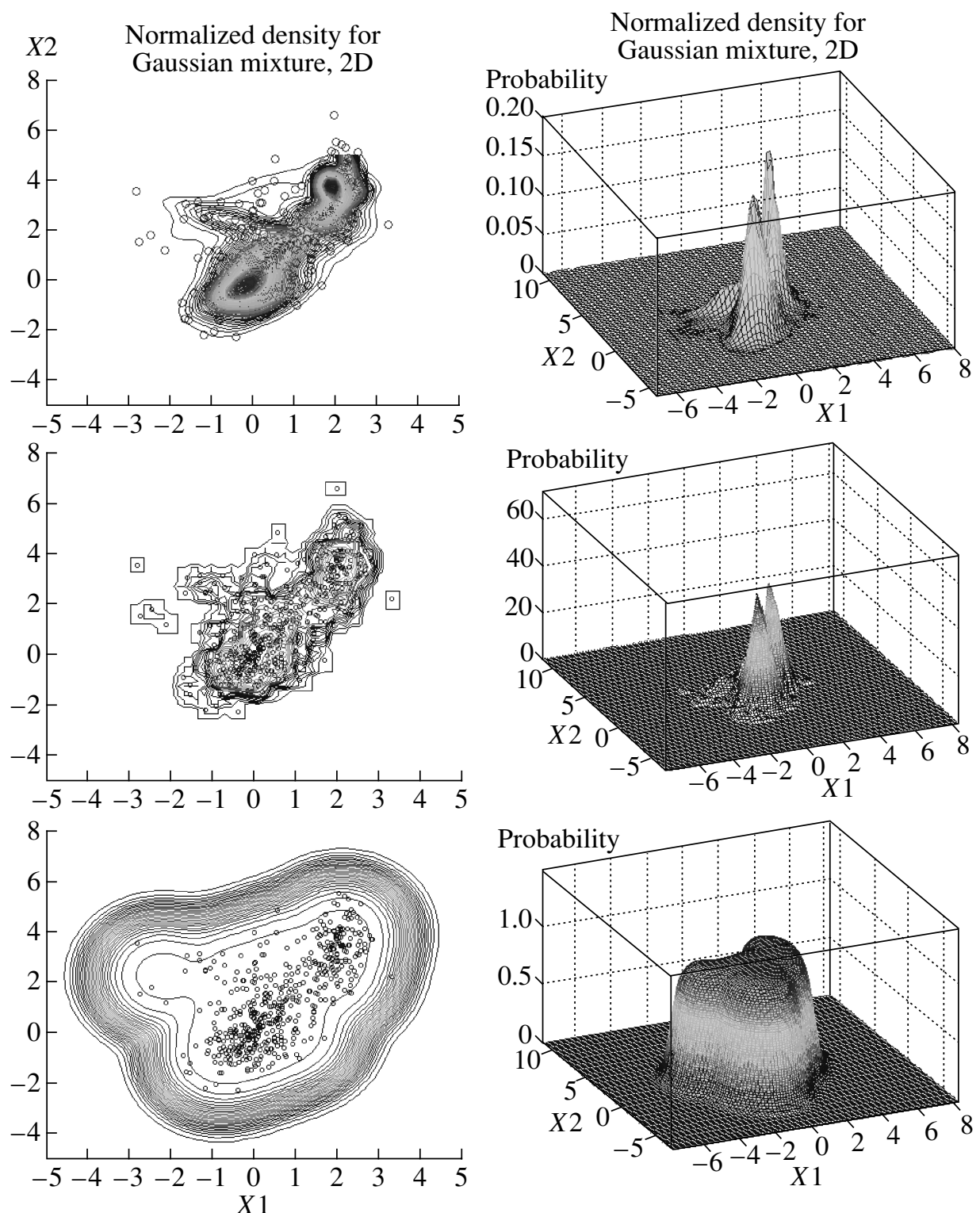

Fig. 5. Comparison of Neural Clouds with other approaches, namely Gaussian mixture and Parzen-window. At the left side $2 \mathrm{D}$ contour lines plots are pictured and at the right—normalized density $3 \mathrm{D}$ plots.

\section{EXAMPLE FOR VIBRATION ANALYSIS}

Numerous papers during the last decades were devoted to the vibration analysis problems [11]. The main idea behind the traditional approaches is that some particular defects generate the noticeable increase of amplitude on the frequencies which could be calculated from e.g. bearing or gearbox parameters. Implementing amplitudes monitoring within some frequency bands around these frequencies, experts determine, whether this situation corresponds to the normal state of the system or to the fault one [12]. The main difficulty of such approach is the following. For the quite complex systems, where the sensors are measuring the superposition of signals from different sources (e.g. system could contain several different bearings, so that their defect frequencies can overlap each other) and the certain noise, standard methods could not be applied. This work is focused on development of an evaluation method, which is aimed to perform a diagnosis of rotating machinery without prior knowledge regarding the internal structure of the system and the system environment. This evaluation method has to be able to distinguish data, obtained in normal situations, from the critical and abnormal ones. 

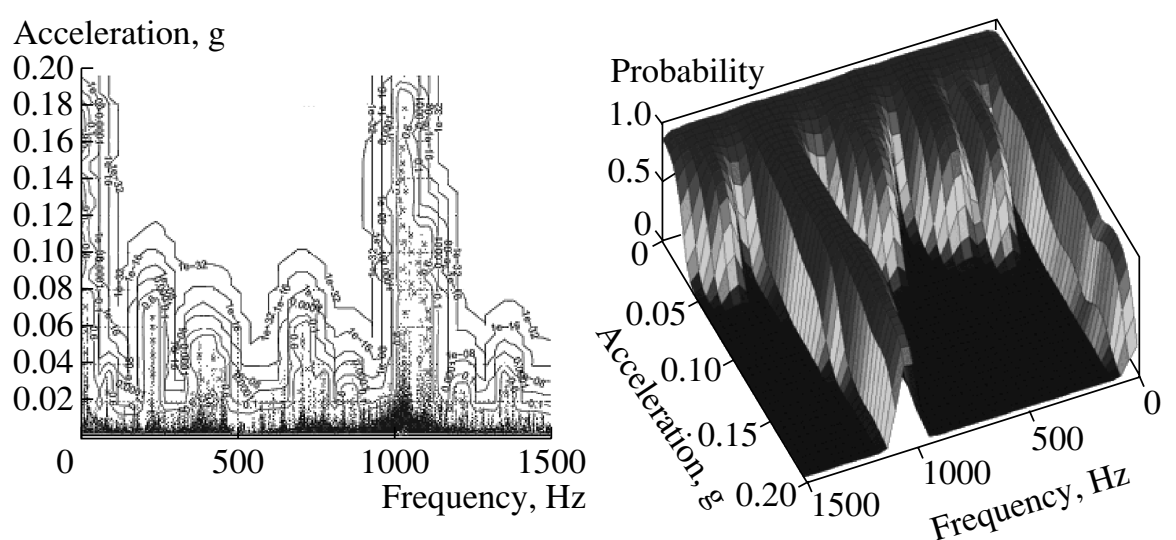

Fig. 6. Spectral data density contours for Fourier spectrum and corresponding 3D surface for the same spectrum which involves visualization of the probability value.

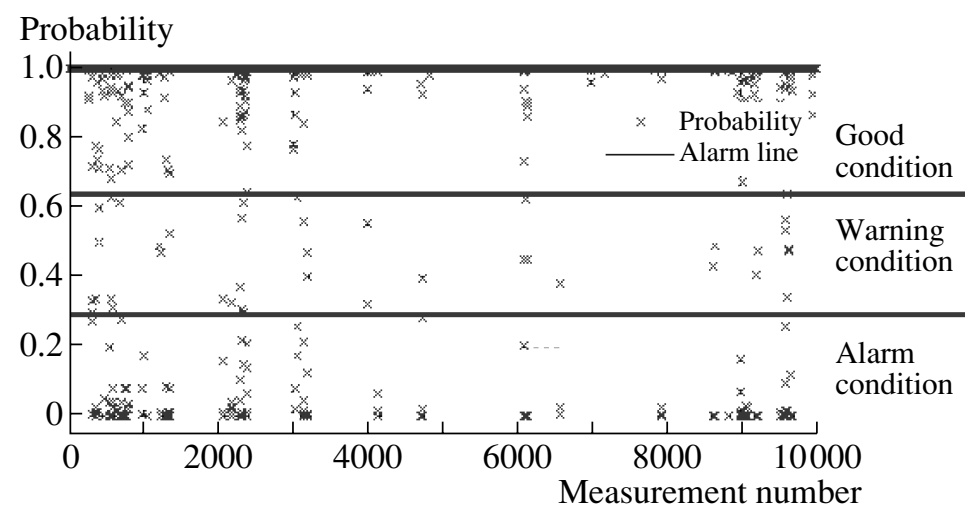

Fig. 7. Confidence along measurements for the detection of failures.

This application of the neuro-fuzzy modeling enables to detect variations from the standard behavior. For this particular application a number of Fourier spectra, measured for the system in normal state, were used for the encapsulating surface (NC) creation. After the so-called training phase, the abovementioned 5 method was used for classification of the succeeding states by computing the confidence values for them.

The spectrum peaks generate different alarm levels, which depend on the corresponding confidence level (see Fig. 6). If there is a critical change in the spectrum, the confidence level decreases from $100 \%$ to $0 \%$. By means of setting the appropriate alarm levels the algorithm (NC) could estimate whether the situation corresponds to the uncritical—good—, critical—warning-, or extremely critical—alarmstates (see Fig. 7).

It represents a model to support the human expert in the task of estimating the remaining system lifetime and preventing the breakdowns. Moreover the evolution of the confidence value could be followed during weeks or months if the $\mathrm{NC}$ is installed as an on-line monitoring system, performing periodic measurements with a frequency of minute order. This represents a significant stage for the concept of the real time diagnosis system. The developed evaluation method enables the detection of system defects and also the efficient diagnostic of the system (which is under monitoring) "health".

\section{EEG CLASSIFICATION PROBLEM}

Modern electroencephalography uses constantly expanding variety of signal processing algorithms. Along with wide developing of computer systems in last decades this makes it possible to extract more information about brain processes in wide range of studies. Numerous papers on quantitative electroencephalography have been published [1-2]. These studies are very important for understanding the nature of processes in human brain. Nevertheless, inverse problem meets many difficulties. In situations, when it is nec- 

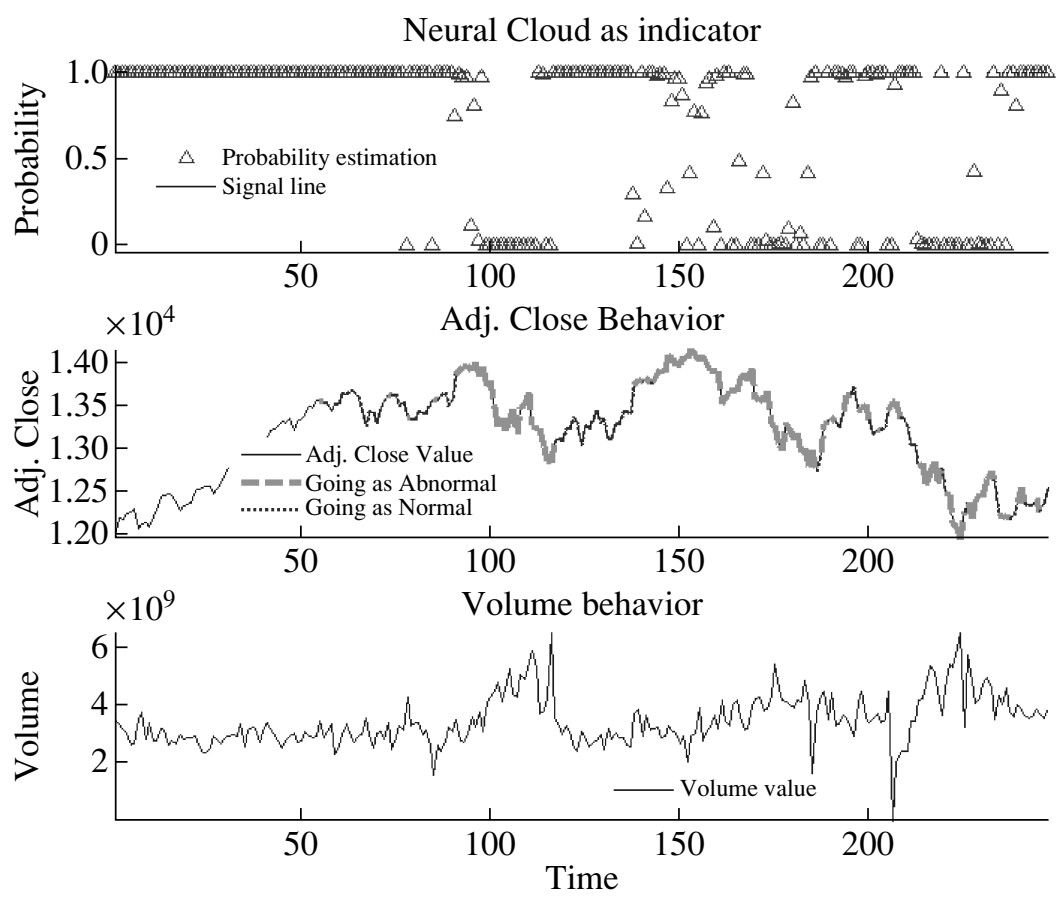

Fig. 8. Generalization set for the NC. Data range for the NC test was from 01.02.2007-01.02.2008. Number of points which do not belong to NC is $47 \%$. Signal line value was chosen to be 0.99 .

essary to make diagnostics of disease, state or peculiarities of the person under study; qualitative methods are still most effective.

The main disadvantage of these methods is the neurophysiologist requirement to make qualified subjective assessment of the EEG recording. In addition, subjective assessment cannot be instant and be used in long time monitoring. One of the possible ways to solve this problem is the usage of intellectual classification systems. Their successful development will make it possible to express diagnostics of brain diseases and building of diagnostic, monitoring systems etc.

Modern diagnostic systems studies, based on artificial neural networks, show the potential of such methods [5-9]. In present work, we suggest a new approach in these methods. In order to show, how powerful the method can be, authors applied this method to the classification of one of the most difficult time series types, namely EEG. Classification of the EEG recordings is very difficult task. The idea behind the classification is to say whether the EEG belongs to healthy subject or not, or to evaluate the state of the subject during the EEG registration. In present only neurologist can firmly identify EEG fragments, which correspond to abnormal patient's state, - - by subjective evaluation.

As the first experience, it was decided to try to distinguish the EEG of the subject with open eyes from the EEG of the same subject with closed eyes. EEG's, recorded in these two states, can be easy distinguished subjectively. We used EEGs, recorded from 19 electrodes, placed according to the international 10-20 system [14]. The idea was to build NC (in 19 dimensional space) for the EEG recording of the man with open eyes and then to present the EEG of the same man with closed eyes to the trained NC in order to obtain the answer, whether the new points belong to the $\mathrm{NC}$ or not, without inducing the prior knowledge about the EEG time series into the algorithm. Results are presented in the Table 1. In the following "training set" means, that some measurements have been randomly taken from the EEG, recorded in the state with open eyes, which was used for the NC creation. Test set stands for the projection of the measurements on the NC, which is not used for NC creation, but which corresponds to the EEG, recorded in the state with open eyes. Generalization set means that the data, which corresponds to the EEG, recorded in the state with closed eyes, have been projected on NC, constructed using the EEG data, which corresponds to the EEG, recorded in the state with open eyes. "Probability" means that with such probability the measurement, projected on NC, belongs to the EEG, recorded in the state with open eyes. For more details about the computational experiment see Table 1.

To make the obtained results more evident, the technique mentioned above has been applied for another dataset. These are EEGs, recorded before and during epileptic seizure. They correspond to two different 
Table 1. EEG in states with open and closed eyes. Results are obtained in computational experiment. The field "Number of AKM centroids" emphasizes the sensitivity of this algorithm with respect to this parameter

\begin{tabular}{|c|c|c|c|}
\hline Parameter description & \multicolumn{3}{|c|}{ Value } \\
\hline $\begin{array}{l}\text { Number of points which do not belong to the NC when the subject closes } \\
\text { eyes (ideal case } 100 \% \text { ) }\end{array}$ & \multicolumn{3}{|c|}{$58 \%$} \\
\hline Number of AKM centroids & \multicolumn{3}{|c|}{53} \\
\hline Computation time to configure NC (CPU: 1.86 Ghz, RAM: 4 GB DDR2) & \multicolumn{3}{|c|}{$25 \mathrm{sec}$} \\
\hline $\begin{array}{l}\text { Evaluation results, number of data point's, which do not belong to NC, ideal } \\
\text { case for training set and test would be } 0 \% \text {, for generalization set } 100 \%\end{array}$ & $\begin{array}{l}\text { Training } \\
\text { set }\end{array}$ & Test set & $\begin{array}{l}\text { Generaliza- } \\
\text { tion set }\end{array}$ \\
\hline & $3.8 \%$ & $7.8 \%$ & $57.6 \%$ \\
\hline
\end{tabular}

Table 2. EEG before and after epileptic seizure. Summary of all results obtained in computational experiment

\begin{tabular}{|c|c|c|c|}
\hline Parameter description & \multicolumn{3}{|c|}{ Value } \\
\hline $\begin{array}{l}\text { Number of points which do not belong to the NC when the subject is in seizure } \\
\text { (ideal case } 100 \% \text { ) }\end{array}$ & \multicolumn{3}{|c|}{$95.7 \%$} \\
\hline Number of AKM centroids & \multicolumn{3}{|c|}{60} \\
\hline Computation time to configure $\mathrm{NC}$ & \multicolumn{3}{|c|}{$65 \mathrm{sec}$} \\
\hline $\begin{array}{l}\text { Evaluation results, number of data point's which do not belong to NC, ideal } \\
\text { case for training set and test would be } 0 \% \text {, for generalization set } 100 \%\end{array}$ & $\begin{array}{l}\text { Training } \\
\text { set }\end{array}$ & Test set & $\begin{array}{l}\text { Generali- } \\
\text { zation set }\end{array}$ \\
\hline & $0.3 \%$ & $0.93 \%$ & $95.7 \%$ \\
\hline
\end{tabular}

states of the brain (in context of epilepsy). These two kinds of EEG recordings also can be easy distinguished visually. Results one can see in the Table 2 below.

As one can see from the results above, this method have easily separated the EEG recordings, made before seizure, from the EEG recordings, made during seizure.

This method may be used in clinical practice-EEG (sometime combined with video-recording) longterm seizure monitoring. This diagnostic procedure makes it possible to record EEG during up to 72 hours $[15,16]$ and is useful when epileptiform activity can be registered only in seizure. Also, in some forms of epilepsy (focal epilepsy, for example) or in absence patient's behavior during seizure may be hardly distinguished from normal, specially if outpatient monitoring (ambulatory EEG monitoring) is used and no medical staff is near by [17]. Application of this method under described circumstances may be very helpful.

\section{EARLY DETECTION OF THE FAILURES OF THE DJIA INDEX}

In order to show, that NC technique is really universal, authors decided to apply NC method for the early detection of the declines on the financial market, since financial market could be considered as a complex system with "normal" and "abnormal" behavior. The idea was to predict recent crashes interpreted as rapidly decays of the Dow Jones Industrial Average (DJIA) index in the period of few days. More specifically the crash on financial market is defined as noticeable decline of indexes or even separate stocks quotations in short period of time. The noticeable decline is interpreted as the price change expressed in $K \%$, where the threshold value $K$ is specific for various financial instruments and even for different time intervals. For example for DJIA index the value of $K$ can be accepted on the level 7-9\% in three days. Since NC method stands for the early detection of the "abnormal" behavior it should extract "abnormal" behavior out of the adjusted close price behavior and volume behavior. There are several works on the prediction of the failures on the financial markets [20-25].

For the back testing of the NC method DJIA dataset in the range from 01.01.2000 till 01.02.2008 was used. The data source used for this problem is http://finance.yahoo.com. In the figure below (see fig. 8) one can find results of such back testing experiment. Authors considered situation on the market as "normal" and trained NC on the data range from 01.01.2000 till 01.02.2007. Then one should apply trained NC for the rest of the data, where few crashes took place. In Fig. 8 one can find results of the application of the NC to the unknown data which contained recent crashes of the DJIA index.

In Fig. 8 (for the middle picture) one can find legend labels marked as "abnormal behavior" and "normal behavior". The behavior was defined from the easy rule. At first one should define "Signal Line" (in the fol- 
lowing signal line was chosen to be 0.99 ) which stands as indicator line. In case "Probability estimation" for the current measurement intersect Signal Line bottom-up one should consider the behavior of the index as "abnormal". In case opposite intersection one should consider the behavior of the index as going to normal or normal.

Overall result is that NC detected abnormal behavior earlier than crashes happened (in average investors would have 3-5 days to make decision before crash). In general such system can be applied for any economical system, which has to be considered as normal or abnormal, for example micro or macro economics can be considered as a complex system with critical phenomena.

\section{CONCLUSIONS}

An efficient one-side classification algorithm has been proposed with some application examples. The main advantage of the presented approach lies in necessity of only one training data set, corresponding to the normal system state. Usually acquisition of such kind data is rather simple, whereas the collection of data related to the fault or abnormal behavior is more difficult.

The overall scheme of NC algorithm usage could be described as selection of the plant conditions set of related measurements, collecting a certain amount of data corresponding to ensured normal state and training of the $\mathrm{NC}$ with this data. After training procedure the $\mathrm{NC}$ ready for the classification of the succeeding states of the system which has to be under monitoring. Whenever some significant changes appear in the system normal state, e.g. due to some environment changes, the NC should be retrained in order to avoid false alarms.

As a possible extension of suggested approach the authors consider the introduction of an automatic procedure for important parameters selection. Here, the noise level of the data as well as the NC sensitivity per input dimension should be provided as hyper-parameters.

The method, presented in the present paper, makes it possible to create the detector of the critical variations in the complex system behavior. This approach was successfully implemented and tested within the real rotating machinery test bench, based on the Siemens automation equipment [13].

\section{ACKNOWLEDGMENTS}

Authors are grateful to N. Koroleva, clinic epileptologist, and Alexander Chukhlovin, neurologist, IHB 6 RAS, for supplying authors with video-EEG recordings from epilepsy patients.

\section{REFERENCES}

1. Sergeev, A., Pavlova, L., and Romanenko, A., Statistical Methods of Human EEG Study, Leningrad: Nauka, 1968.

2. Evans, J. and Abarbanel, A., Introduction to Quantitative EEG and Neurofeedback, New York: Academic Press, 1999.

3. Schlang, M., Lang, B., Poppe, T., Runkler, T., and Weinzierl, K., Current and Future Development in Neural Computation in Steel Processing, Control Engineering Practice, 2001, vol. 9, pp. 975-986.

4. Mokhov, I. and Minin, A., Advanced Forecasting and Classification Technique for Condition Monitoring of Rotating Machinery, Proceedings of the 8th International Conference On Intelligent Data Engineering and Automated Learning (IDEAL'07) (Birmingham, UK, December 16-19, 2007, Springer), pp. 37-46.

5. Sinha, K., Artificial Neural Network Detects Changes in Electro-Encephalogram Power Spectrum of Different Sleep-Wake States in an Animal Model of Heat Stress, Medical and Biological Engineering and Computing, September 2003, vol. 41, no. 5, pp. 595-600.

6. Duta, M., Alford, C., Wilson, S., and Tarassenko, L., Neural Network Analysis of the Mastoid EEG for the Assessment of Vigilance, International Journal of Human-Computer Interaction, 2004, vol. 17, no. 2, pp. 171-199.

7. Karayiannis, N., Mukherjee, A., Glover, J., Ktonas, P., Frost, J.Jr., Hrachovy, R., and Mizrahi, E., Detection of Pseudosinusoidal Epileptic Seizure Segments in the Neonatal EEG by Cascading a Rule-Based Algorithm with a Neural Network, IEEE Transactions on Biomedical Engineering, 2006, vol. 53, no. 4, pp. 633-641.

8. James, J., Jones, D., Bones, J., and Carroll, J., Detection of Epileptiform Discharges in the EEG by a Hybrid System Comprising Mimetic, Self-Organized Artificial Neural Network, and Fuzzy Logic Stages, Clinical Neurophysiology, 1, December 1999, vol. 110, no. 12, pp. 2049-2063 (15).

9. Tsuji, Y., Usui, T., Sato, Y., and Nagasawa, K., Development of Automatic Scoring System for Sleep EEG Using Fuzzy Logic, Journal of Robotics and Mechatronics, 1993, vol. 5, no. 3, pp. 204-208.

10. Bishop, C., Pattern Recognition and Machine Learning, Springer, 2006.

11. Barkova, N.A., The Current State of Vibroacoustical Machine Diagnostics, http://www.vibrotek.com/articles.php. 
12. Barkov, A.V., Barkova, N.A., and Mitchell, J.S., Condition Assessment and Life Prediction of Rolling Element Bearings, Sound and Vibration, June 1995, pp. 10-17; September, pp. $27-31$.

13. CASTOMAT Diagnostic Systems, http://www.siemens.com/castomat

14. Jasper, H., Report of the Committee on Methods of Clinical Examination in Electroencephalography, EEG clin. Neurophysiol, 1958, vol. 10, pp. 370-375.

15. Lagerlund, T.D., Cascino, G.D., Cicora, K.M., and Sharbrough, F.W., Long-Term Electroencephalographic Monitoring for Diagnosis and Management of Seizures, Mayo Clin. Proc., 1996, pp. 1001-1006.

16. Legatt, A.D. and Ebersole, J.S., Options for Long-Term Monitoring, in Epilepsy: A Comprehensive Textbook, Engel, J., Pedley, T.A., Eds., vol. 1. Philadelphia: Lippincott Williams and Wilkins; 1998, pp. 1001-1020.

I 17. Dericio@glu, N., Albakir, M., and Saygi, S., The Role of Patient Companions in Long-Term Video-EEG Monitoring, Seizure, 2000, Mar, vol. 9, no. 2, pp. 124-127.

18. Wikipedia Internet Portal, http://en.wikipedia.org/wiki/Parzen_window

19. Wikipedia Internet Portal, http://en.wikipedia.org/wiki/Mixture_model

20. Sornette, D., Why Stock Markets Crash, Princeton and Oxford: Princeton University Press, 2003.

21. Johansen, A., Sornette, D., and Ledoit, O., Crashes as Critical Points, Int. J. Theor. and Appl. Finance, 2000, vol. 3, no. 2, pp. 219-255.

22. Sornette, D., Critical Market Crashes, Physics Reports, 2003, vol. 378, pp. 1-98.

23. Agaev, I.A. and Kuperin, Yu.A., Multifractal Analysis and Local Hoelder Exponents Approach to Detecting Stock Markets Crashes, http://xxx.lanl.gov/ftp/cond-mat/papers/0407/0407603.pdf, 2004.

24. Sornette, D., Johansen, A., and Bouchaud, J.-P., Stock Market Crashes, Precursors and Replicas, J. Phys. I France, January, 1996, vol. 6, pp. 167-175.

25. Kuperin, Yu.A. and Schastlivtsev, R.R., Modified Holder Exponents Approach to Prediction of the USA Stock Market Critical Points and Crashes, 15p., arXiv: 0802.4460, physics. soc-ph, 2008, http://xxx.lanl.gov

SPELL: 1. multi-dimensional, 2. centroids, 3. centre, 4. encapsulator, 5. abovementioned, 6. epileptologist 